\title{
ANALYSE ECONOMIQUE ET PRINCIPE DE RATIONALITÉ
}

Chacun sait que ceux qui pratiquent les sciences humaines sont souvent amenés à se tourner vers les sciences physiques pour y chercher la clé qui permettrait d'assurer à leur propre démarche le type de respectabilité scientifique dont ces dernières ont bénéficié de plus en plus depuis le $\mathrm{XVII}^{\mathrm{e}}$ siècle ${ }^{1}$. Pourtant cette émulation, toute saine et légitime qu'elle soit, a engendré une profonde frustration dans la mesure où, comme on aime à le rappeler, les sciences humaines s'intéressent par définition à des actions « humaines » dont le caractère apparemment libre et spontané semble incompatible avec l'établissement de ces lois scientifiques qui confèrent aux sciences physiques à la fois leur enviable respectabilité et leur étonnant pouvoir de prédiction. Ainsi, dans la mesure du moins où ils se posent des questions d'ordre méthodologique, les praticiens des sciences humaines se sont vus contraints de tenir compte du problème posé à l'analyse scientifique par ce caractère libre et spontané des actions humaines.

Bien sûr, rien n'oblige un praticien des sciences humaines à se prononcer sur la question métaphysique, longuement débattue par les philosophes de tous les âges, concernant les rapports de la liberté humaine et du déterminisme. Son intérêt en la matière est strictement méthodologique; mais pourtant, si, pour être en mesure d'arriver à des résultats scientifiques concluants, il décide de traiter les êtres humains comme des choses soumises au même titre que les autres à des lois scientifiques, sa prise de position méthodologique n'en équivaut pas moins à une prise de partie dans ce débat. Aussi, pour résoudre cette tension entre un idéal causaliste et déterministe emprunté aux sciences physiques et un objet d'étude qui semble se prêter assez mal à une telle approche, les praticiens

1. L'auteur tient à remercier Éric Brian, Stéphan D'Amour, Philippe Mongin et Robert Nadeau de leurs utiles suggestions ainsi que le C.R.S.H.C. (Ottawa) et le Fonds F.C.A.R. (Québec) de leur assistance financière. 
des sciences humaines ont généralement adopté l'une ou l'autre des trois voies suivantes.

La première de ces voies a consisté à nier la liberté des actions humaines ou, en tout cas, à nier que le trait ainsi désigné empêche de soumettre ces actions à une analyse résolument déterministe. La deuxième a consisté à refuser d'analyser les actions humaines individuellement et à s'en tenir plutôt à un niveau d'agrégation tel que les phénomènes globaux qui y sont observés peuvent faire l'objet d'un déterminisme qui n'affecte pas, en tant que telles, les actions individuelles dont ils résultent. La troisième voie, enfin, a consisté à prendre les actions humaines individuelles pour objet d'étude en cherchant à surmonter les difficultés engendrées par l'acceptation de leur caractère libre et spontané. Toutefois, pour ne pas voir un tel objet d'étude échapper littéralement à leur prise, ceux qui ont opté pour cette dernière voie devaient postuler que les actions humaines, toutes libres et spontanées qu'elles puissent être, ne sont pas arbitraires pour autant, dans la mesure où elles sont adaptées à des fins, bref dans la mesure où elles sont des actions rationnelles.

DÉTERMINISME CAUSAL OU ANALYSE STATISTIQUE

Avant d'examiner plus attentivement les problèmes posés par cette troisième voie et par le postulat de rationalité qui en est la condition fondamentale, voyons un peu ce qu'il en est des deux premières.

Adopter la première voie caractérisée sommairement ci-dessus, c'est en somme nier la spécificité épistémologique des sciences humaines et soutenir que les actions humaines sont soumises à des lois causales au même titre que les comportements animaux et que les phénomènes physiques en général. Une science humaine, qui aurait ainsi toutes les caractéristiques des sciences physiques, pourrait permettre de substituer des prédictions exactes aux vagues considérations inspirées par une sagesse traditionnelle, respectable, certes, mais incapable de progrès. Cette ambition, esquissée dès le XviII ${ }^{e}$ siècle mais affirmée surtout au $\mathrm{xIx}^{\mathrm{e}}$ siècle $^{2}$, explique l'intérêt suscité par les diverses philosophies déterministes qui ont cherché à tirer parti des découvertes biologiques et surtout neurologiques

2. Pour un aperçu des conceptions qu'on se faisait, au Xvir ${ }^{e}$ et au xIX ${ }^{e}$ siècle, des sciences humaines et de leurs relations aux sciences physiques, on consultera utilement l'ouvrage classique de Georges GusDorf, Introduction aux sciences humaines, essai critique sur leurs origines et leur développement, Paris, Ophrys, 1974 ( $1^{\mathrm{r}}$ éd., 1960). 
pour enraciner l'action humaine dans un univers physique et causal. Au $\mathrm{xx}^{\mathrm{e}}$ siècle, cette tendance a connu des succès indiscutables avec le développement de la psychologie behavioriste ${ }^{3}$, mais les sciences sociales en tant que telles, comme la sociologie, l'économie ou l'histoire, ne pouvaient s'y rallier que beaucoup plus difficilement dans la mesure où les problèmes posés par la réduction du social au psychologique se seraient ajoutés pour elles à ceux, déjà fort ardus, posés par la réduction du psychologique au biologique. D'ailleurs, pour plusieurs théoriciens des sciences humaines, incluant cette fois la psychologie, vider le comportement humain de toute trace de liberté et de spontanéité a paru être un prix trop élevé à payer pour la satisfaction de détenir une science humaine bénéficiant des avantages incontestables du déterminisme causal. C'est pourquoi, sans cesser pour autant de lorgner vers les sciences physiques quand ils ont à préciser ce qui caractérise la démarche scientifique, les praticiens des sciences humaines, et les économistes en particulier, ont été amenés, le plus souvent, à emprunter d'autres voies, mieux adaptées à leur objet d'étude, mais moins susceptibles de leur apporter la caution qu'aurait pu leur procurer une application plus servile des méthodes mises au point par ces sciences physiques.

La deuxième voie évoquée plus haut a l'avantage de ne pas supposer formellement résolu le débat philosophique à propos de la spécificité des actions humaines. Elle consiste à concentrer l'analyse scientifique sur les phénomènes globaux dont l'évolution peut être rigoureusement déterminée et soumise à des lois même quand les actions humaines dont ces phénomènes résultent sont présumées libres et spontanées. Soutenir que, ceteris paribus, le taux de suicide varie avec l'heure du jour ou que le taux de chômage varie en raison inverse du taux d'inflation n'est pas soutenir que les individus qui se suicident ou que les patrons qui embauchent ou congédient des employés sont mus par des mécanismes neurologiques irrésistibles. L'individu le plus désespéré pourrait décider librement de ne pas se suicider ou de le faire à une heure jugée peu propice à ce genre de décision et le patron le plus impitoyable pourrait, au moment où le taux d'inflation est au plus bas, décider librement d'accroître l'embauche de nouveaux travailleurs. Tout ce qui est impliqué par cette approche, c'est que, quel que soit le degré de liberté de ceux qui s'engagent dans de telles actions, quand on considère l'ensemble d'une société, une tendance se manifeste statistiquement avec une régularité suffisante pour que l'on puisse parler de loi. Faute de pouvoir prédire le comportement des individus en cause, on peut espérer fonder une science de grands

3. Pour une intéressante analyse épistémologique de cette tendance, on lira : Alexander Rosennerg, Philosophy of Social Science, Boulder, Westview Press, 1988, chap. 3. 
ensembles dont on pourrait au moins prédire les mouvements de manière statistique. Favorisée par la plupart des sociologues et par les économistes qui s'adonnent à la macro-économie, cette approche n'a pas manqué d'attirer les historiens qui ont vu dans l'histoire quantitative des grands ensembles une voie susceptible d'accroître le caractère scientifique d'une discipline que l'on aurait tort de trop associer à la singularité et à l'unicité des événements.

Cette voie, d'ailleurs, est d'autant plus séduisante que la science physique elle-même, depuis le début du $x x^{e}$ siècle s'est, en quelque sorte, redéployée à partir de bases purement statistiques. Toutefois, une chose demeurait assez gênante dans l'adoption systématique d'une telle démarche. On pouvait difficilement reprocher aux physiciens de ne pouvoir rendre compte des mouvements individuels de particules infinitésimales dont l'existence même, sans leurs théories, serait demeurée insoupçonnée, dans la mesure où, en procédant ainsi, ils pouvaient expliquer et prédire avec une précision remarquable à peu près tous les phénomènes physiques qu'il nous est donné d'observer. Dans les sciences humaines, par contre, les phénomènes les plus immédiatement observables sont justement les comportements individuels des êtres humains et, puisqu'ils constituent, en un sens, l'objet même des sciences humaines, il est assez gênant de devoir renoncer à les expliquer. Sans doute est-ce déjà beaucoup pour un économiste de pouvoir prédire (du moins au sens où il assure que ces événements sont fortement probables) que, dans un pays donné, le taux de chômage et le taux d'inflation évolueront en des sens opposés ou que le taux de chômage grimpera au cours des prochains mois ; mais puisque cette hausse du taux de chômage est forcément due à des événements comme le fait que divers entrepreneurs aient décidé de congédier des employés ou que certaines personnes aient pris la résolution de rechercher un emploi, il parait légitime de chercher à expliquer ce qui se passe au niveau des décisions individuelles elles-mêmes. Mais à ce niveau, on se heurte à nouveau au conflit du déterminisme et du caractère libre et spontané des actions humaines. Comme on ne peut tout de même pas soutenir que, face à une baisse de leurs profits, les entrepreneurs sont mus par une force irrésistible, de nature hormonale ou autre, qui les amène à congédier un certain nombre d'employés, il faut admettre que ces entrepreneurs sont des êtres libres qui, en ces circonstances, ont le pouvoir d'agir de toutes sortes de façons. Mais alors il paraît bien difficile de prédire leur comportement; et si tel est le cas, peut-on encore prétendre que l'on peut, en l'expliquant, rendre compte des conséquences éventuelles d'un tel comportement? 
C'est pourtant à ce problème que la troisième voie adoptée par nombre de praticiens des sciences humaines prétend apporter une solution. La clé de cette solution tient au fait que l'on peut supposer que les entrepreneurs en question sont des individus rationnels et que, face à une situation donnée, un individu rationnel adopte librement la solution qu'il juge la plus susceptible de lui permettre d'atteindre ses fins. L'intérêt d'une telle solution, c'est qu'elle permet, sans remettre en question la liberté et la spontanéité des actions humaines - sans faire de l'entrepreneur une sorte d'automate - de prédire ce que seront ces actions ou, en tout cas, de les expliquer, pour peu que l'on suppose connues les fins poursuivies par les individus d'une certaine catégorie. Si l'on suppose, par exemple, que le but des entrepreneurs est d'accroittre les profits de leur entreprise, il sera possible en principe de prédire ce que, dans des circonstances données, ils choisiront (rationnellement mais librement) de faire et d'expliquer ainsi ce qu'ils ont fait à partir de ces circonstances. Et si, sur cette base, on construit une théorie rendant compte avec suffisamment de rigueur des conséquences de telles actions, on pourra alors soutenir qu'on est parvenu à fournir une explication "scientifique " de ces phénomenes sociaux ou, du moins, une explication du type de celles qu'on est en droit d'attendre des sciences humaines.

Le postulat de rationalité constitue donc bien la clé de toute explication de ce type. Le comportement n'est expliqué que dans la mesure où l'on postule qu'il s'agit d'un comportement rationnel, c'est-à-dire d'un comportement adapté à une fin dont on peut faire état pour en rendre compte. C'est ainsi que ce postulat, qu'on présente souvent comme l'application d'un principe de rationalité ${ }^{4}$, a joué un rôle fondamental dans l'analyse micro-économique marginaliste qui, depuis les années 1870 , a constitué sans conteste le paradigme d'une telle approche des sciences humaines. Les particularités de cette approche n'ont toutefois pas enlevé aux économistes marginalistes l'ambition de construire des modèles théoriques comparables à ceux de leurs collègues physiciens et de soumettre ensuite à des tests les prédictions dérivées de ces modèles. S'ils pouvaient espérer obtenir un certain succès (qu'il ne s'agit toutefois pas d'évaluer ici) dans ces prédictions, c'est évidemment parce que le comportement humain manifeste une certaine régularité ou, si l'on

4. Bien que «postulat de rationalité et « principe de rationalité $»$ soient souvent considérés comme des expressions interchangeables, je préfère employer respectivement l'une ou l'autre selon que l'accent est mis davantage sur l'aspect conventionnel du « postulat \% ou sur le « principe $»$ qu'on croit pouvoir invoquer pour le fonder. 
préfère, obéit à certaines lois. Mais rien n'oblige à supposer que ces lois soient des lois causales comme celles qui régissent les phénomènes physiques; il suffit, en effet, de postuler que les agents économiques sont rationnels pour qu'il soit possible de prédire, jusqu'à un certain point, leur comportement et, ce faisant, de l'expliquer.

On voit donc en quel sens le principe de rationalité peut être considéré comme le postulat fondamental de la micro-économie, mais on doit reconnaître que le recours à ce postulat pose de sérieux problèmes épistémologiques. Il suffit, pour s'en convaincre, de réfléchir au fait qu'on a de bonnes raisons de soutenir que ce principe est faux. Au nom de quoi, en effet, peut-on affirmer que les individus, qu'ils soient entrepreneurs ou simples consommateurs, agissent de façon rationnelle ? Les exemples de comportements irrationnels ne sont-ils pas trop constants autour de nous pour que l'on puisse vraiment prendre au sérieux une science qui reposerait sur un postulat de rationalité ? Les critiques de l'économie, en tout cas, n'ont pas manqué de faire de ce constat une arme qu'ils ont inlassablement dirigée contre les prétentions à la scientificité des économistes 5 .

Pour qui entend analyser le comportement économique selon la troisième voie ici décrite, le spectre de l'irrationalité paraît, d'ailleurs, beaucoup plus menaçant que la simple objection de ceux qui, au nom de leurs conceptions humanistes, s'offusquent des moindres tentatives d'étendre l'analyse scientifique à l'univers des actions humaines qui sont présumées libres et spontanées. Pour rendre compatible la liberté et le déterminisme requis par l'analyse scientifique, les praticiens des sciences humaines n'ont, en un sens, qu'à parodier saint Augustin en proposant, en quelque sorte, aux agents économiques le pacte suivant: "Sois rationnel et choisis ce que tu veux. "Peu importe, en effet, que les choix des êtres humains soient métaphysiquement libres, peu importe, en somme, que ceux qui prennent une décision détiennent par devers eux une sorte de pouvoir contrefactuel de choisir autre chose que ce qu'ils choisissent effectivement, s'ils sont rationnels, s'ils se laissent guider par la raison, ils feront un choix qui pourra, en principe, être prédit et expliqué par des analystes qui, partageant avec eux cette rationalité, savent ce qu'il est rationnel de faire dans les circonstances où ils se trouvent.

$\mathrm{Si}$, par contre, on admet qu'ils peuvent ne pas être rationnels et ne pas se laisser guider par la raison, alors c'est la possibilité mếme d'élaborer

5. Les critiques de Lester contre la maximisation du profit, en part. in Richard A. LESTER, « Shortcomings of Marginal Analysis for Wage-Employment Problems », American Economic Review, 36, $I, 1946$, p. 37-60, ont été au cour d'une vive polémique dont on pourra lire une intéressante analyse critique in Philippe Mowalw, \& La controverse sur l"entreprise (1940- 
une micro-économie ou une science humaine du même type qui semble s'effondrer. Quant aux macro-analyses, qui s'en tiennent à rendre compte des mouvements d'ensemble sans chercher à les expliquer à partir des comportements individuels eux-mêmes, elles ne s'en porteraient pas tellement mieux, car si on peut prédire que l'évolution du taux d'inflation affectera celle du taux de chômage, c'est qu'on suppose que, dans leur ensemble, les consommateurs et les entrepreneurs réagissent de façon relativement constante à une hausse ou à une baisse des prix, de la demande ou des possibilités de crédit. On pourrait objecter que le seul fait que nombre de comportements soient irrationnels n'a rien de très dévastateur pour une science humaine puisque, dans la mesure où ils consistent souvent en des réactions quasi mécaniques, les comportements irrationnels n'en sont que plus aisément prévisibles. Rien n'est plus irrationnel que la superstition; or si j'offre à une personne superstitieuse de choisir entre deux billets d'avion dont l'un est en date d'un vendredi 13, je peux, sans grand risque d'erreur, prédire qu'elle choisira l'autre, alors que si je faisais la même offre à un individu nullement superstitieux parce que plus « rationnel », ma prédiction risquerait d'être parfaitement arbitraire. Ceci parait incontestable, mais c'est en un sens différent qu'il faut comprendre ici la notion d'irrationalité. Le comportement superstitieux est un comportement tout aussi constant que le comportement rationnel et c'est pour cela que la prédiction peut y trouver prise. La forme d'irrationalité qui est désastreuse pour les sciences humaines est celle qui est associée à un comportement inconstant, fugitif ou purement fantaisiste. Bien sûr, le comportement rationnel n'est pas un comportement rigide, mais il est un comportement adapté à la situation qui prévaut et c'est parce qu'il s'y adapte constamment qu'on peut le qualifier de constant. À l'inverse, un comportement irrationnel est un comportement inadapté à la situation parce que rebelle à toute règle et à toute forme d'adaptation ; bref, le comportement irrationnel par excellence est le comportement gratuit. Peut-être André Gide voulait-il, plus ou moins consciemment, embarrasser son oncle Charles, le grand économiste, quand, dans ses romans, il s'ingéniait tant à imaginer des " actes gratuits ". Quoi qu'il en soit, la possibilité que les êtres humains puissent agir de façon gratuite, c'est-à-dire n'avoir cure d'adapter leurs actes à quoi que ce soit ou d'être constant en quoi que ce soit, semble devoir invalider le postulat de rationalité et, avec lui, l'entreprise scientifique qui cherchait à y prendre appui. Sans doute, les actes gratuits assez spectaculaires de certains héros de Gide ne sont-ils pas très représentatifs du comportement humain,

1950) et la formation de l'"irréalisme méthodologique" ", Économies et sociétés, t. XX, mars 1986, p. 95-151. 
mais si le comportement effectif des êtres humains se situe quelque part entre ces deux extrêmes que sont la parfaite rationalité postulée par les économistes et la parfaite gratuité imaginée par Gide, c'en est fait du postulat de rationalité, du moins en tant que principe universel pouvant servir de base à une micro-économie scientifique. Pour qu'un tel principe soit disqualifié, il n'est pas nécessaire que les êtres humains ne soient nullement rationnels, il suffit qu'ils ne le soient pas constamment et que, par conséquent, il ne soit plus possible de prédire leur comportement en postulant qu'ils le sont.

TROIS OPTIONS POSSIBLES FACE AU PRINCIPE DE RATIONALITÉ

Cette difficulté épistémologique majeure ne pouvait apparemment être surmontée que de trois façons par les défenseurs de la micro-économie, science humaine qui a le plus, et de loin, cherché à tirer parti du postulat de rationalité. (1) Ils pouvaient renoncer carrément au postulat de rationalité et chercher à fonder leurs conclusions scientifiques sans son secours, (2) ils pouvaient soutenir que la validité du postulat de rationalité importe peu puisque sa fausseté éventuelle ne gênerait en rien le caractère scientifique d'une démarche qui se mesurerait moins à la vérité d'une théorie qu'à sa capacité de prédire, (3) ils pouvaient chercher à montrer que, malgré les apparences qui semblent établir le contraire, le postulat de rationalité est parfaitement adapté au développement d'une théorie scientifique. Il paraît donc indiqué de s'arrêter brièvement sur chacune de ces trois options - et particulièrement sur la troisième qui consiste justement à tirer parti du postulat de rationalité - en vue de faire ressortir l'importance décisive đu principe de rationalité (qui légitime ce postulat) et la fonction inédite qui lui est dévolue en sciences humaines.

La première de ces trois options - la mise au rancart du postulat de rationalité - ne semble pas avoir beaucoup retenu l'attention des économistes. Il est vrai que l'économiste Gary Becker s'est audacieusement engagé sur cette voie dans un texte fort ingénieux ${ }^{6}$ qui visait à montrer qu'il est possible d'établir les principaux théorèmes de la micro-économie même quand on suppose que les agents économiques se comportent de façon parfaitement irrationnelle. Toutefois, ce texte, qui a fait l'objet de

6. Gary S. BeCKer, «Irrational Behavior and Economic Theory», Joumal of Political Economy, LXX, 1, 1962, p. 1-13. 
vives critiques, ne semble pas avoir eu beaucoup de suite, même chez son auteur dont les travaux ultérieurs ont fait abondamment appel au postulat de rationalité. Au terme du débat bref et peu remarqué mais d'un grand intérêt théorique que cette tentative a suscité, on peut conclure qu'elle équivalait, comme j'ai essayé de le montrer ailleurs ', à l'abandon pur et simple du mode d'explication fondé sur la rationalité qui caractérise la troisième voie présentement examinée au profit du mode d'explication fondé sur un mécanisme automatique et donc sur un déterminisme causal analogue à celui que requérait la première voie discutée plus haut.

Si cette première option n'a pas vraiment été retenue par les économistes, la deuxième option, par contre, semble bien être celle qui a paru la plus séduisante à leurs yeux, depuis que l'un d'entre eux, Milton Friedman, s'en est fait le défenseur dans son célèbre article de $1953^{8}$. La thèse de Friedman, souvent qualifiée d'instrumentaliste, reposait sur l'idée que le seul critère permettant de reconnaitre la scientificité d'une démarche explicative doit résider dans la validité des prédictions qu'elle autorise. Aussi, aux yeux de Friedman, un postulat comme le postulat de rationalité, dans la mesure où il n'est pas lui-même une prédiction, n'a pas à être testé; peu importe même qu'il soit faux, s'il joue un rôle instrumental dans l'établissement de prédictions qui, elles, sont testées avec succès, il aura rendu possible une analyse positive et scientifique dont il constituera, par le fait même, une partie intégrante. Cette forme d'instrumentalisme appliqué à l'économie a toutefois suscité de nombreuses oppositions chez les méthodologues de cette discipline, tant à propos du bien-fondé de cette approche épistémologique qu'à propos du fait qu'elle présuppose l'existence de tests qui, à vrai dire, demeurent assez peu concluants en économie. Si la première des trois options examinées consistait à rejeter carrément le principe de rationalité, cette deuxième option consiste à en réduire l'importance en le confinant à un rôle purement instrumental plutôt qu'explicatif. Aussi, même si la thèse de Friedman a donné lieu à l'un des débats les plus soutenus et les plus importants de la méthodologie économique ${ }^{9}$, il ne saurait être question d'en examiner les tenants et aboutissants, puisqu'il s'agit justement ici de voir en quel sens le principe de rationalité constitue bel et bien l'élément cen-

7. Maurice LagueUx, « Kirzner vs Becker : Rationality and Mechanisms in Economics », in Perspectives on the History of Economic Thought, Aldershot (Hants, G.-B.), Edward Elgar Publishing, 1993.

8. Milton Friedman, "The Methodology of Positive Economics", in M. FrIedman, Essays in Positive Economics, Chicago, The University of Chicago Press, 1953, p. 3-43.

9. La seule liste des participants à ce débat étant trop longue à établir, je me contenterai de renvoyer à ma propre contribution qui vise elle-même à en expliquer le caractère équivoque : M. LAGUEUX, "Friedman's "Instrumentalism" and Constructive Empiricism in Economics $"$, à paraître dans Theory and Decision. 
tral d'un type d'explication scientifique dont les caractéristiques, il est vrai, restent à dégager et le bien-fondé à établir.

En assurant contre vents et marées que le postulat de rationalité est parfaitement adapté au développement des sciences humaines, les défenseurs de la troisième option évoquée ci-dessus mettent justement en relief la spécificité du mode d'explication qui y prend appui, mais cette troisième approche semble poser au moins autant de problèmes que les deux premières. Comme on l'a signalé plus haut, il paraîtrait assez singulier de soutenir sérieusement que les individus agissent toujours rationnellement quand il semble si facile d'accumuler des exemples de comportements irrationnels. Pourtant, tout dépend de ce que l'on considère rationnel. Certes, le terme « rationnel » évoque, on l'a vu également, l'idée d'adaptation à des fins, de telle sorte qu'une action humaine sera dite rationnelle si elle est adaptée aux fins ou aux buts que poursuit son auteur. Mais quels sont ces buts? En un sens, seule la personne qui les poursuit peut répondre à cette question ; mais s'il en est ainsi, comment une action pourrait-elle être considérée irrationnelle?

UNE ACTION PEUT-ELLE ÉTRE IRRATIONNELLE?

Si je rêve de m'acheter un jour une maison et que je refuse un emploi supplémentaire fort rémunérateur que vous $m$ 'offrez, vous pouvez estimer que mon refus est irrationnel compte tenu du but que je poursuis, mais je peux rétorquer que mon choix est, au contraire, tout à fait rationnel car mon but est de mener une vie gouvernée par la jouissance d'un loisir raisonnable aujourd'hui et celle de la possession d'une maison demain. Il suffit que j'estime possible d'acheter en temps et lieu la maison de mes rêves sans être obligé de me priver présentement de tout loisir pour que mon choix puisse être qualifié de parfaitement adapté à mes buts et donc de parfaitement rationnel. Mais si quelques années plus tard, je me rends compte que j'avais surestimé mes chances d'être en mesure d'acheter cette maison et si, en conséquence, je me mords alors les doigts de n'avoir pas, en temps et lieu, accepté cet emploi supplémentaire qui m'aurait sans doute permis d'accumuler le capital requis pour assurer cette transaction, devrait-on en conclure que ma décision de refuser cet emploi était irrationnelle?

Deux raisons laissent penser qu'il serait pour le moins hasardeux de le faire. D'une part, admettre que, faute d'informations suffisantes sur l'évolution du prix des maisons et des taux hypothécaires, mon estima- 
tion d'alors ait été mauvaise n'est pas admettre qu'elle ait entraîné une décision irrationnelle. S'il fallait, en effet, qualifier d'irrationnelles toutes les décisions qui ne sont pas parfaitement adaptées parce qu'elles reposent sur une information insuffisante, la rationalité supposerait l'omniscience car tous les événements futurs peuvent, en principe, affecter les conséquences d'une décision. Aussi vaut-il mieux qualifier de rationnelles les décisions qui paraissent adaptées à la lumière des seules informations disponibles, au moment où elles sont prises, à la personne qui doit les prendre. D'autre part, un certain nombre d'années après avoir dû renoncer à mon rêve, je pourrais bien en arriver à la conclusion que, étant donné la brièveté de l'existence humaine, une vie consacrée à l'acquisition de biens matériels est une vie perdue et que le loisir dont j'ai pu bénéficier était, après réflexion, beaucoup plus valable que la maison que j'aurais pu me procurer en le sacrifiant. Faudrait-il alors réviser $a$ nouveau le verdict et décréter que ma décision première était bel et bien rationnelle? Mais au nom de quoi pourrions-nous soutenir que ce sont mes perceptions en ce troisième temps et non celles qui prévalaient au moment de mon remords qui devraient servir de critère à l'évaluation d'une décision prise à un moment antérieur à ces deux-là ? Bref, les perceptions - qu'elles soient l'expression de désirs profonds ou qu'elles résultent des seules informations disponibles à un moment donné - se modifient constamment avec le temps, de telle sorte qu'il vaut mieux qualifier de rationnelles les décisions qui paraissent adaptées à la lumière des seules perceptions qui s'appuient sur des informations disponibles et qui reflètent des désirs profonds au moment où elles sont prises. Mais s'il en est ainsi, il semble bien qu'on peut conclure que toute action est rationnelle, puisque la décision qui parait la plus stupide ne peut être prise que parce que la personne qui la prend y voit, compte tenu des informations qui lui sont alors disponibles, une façon de satisfaire le désir, si fugitif qu'il soit, qui l'anime à cet instant précis. Même les actes gratuits décrits par Gide pourront être qualifiés de rationnels, puisqu'ils peuvent être compris comme des réponses parfaitement adaptées à la réalisation des objectifs de personnes qui estiment important à un moment donné de briser la triste atmosphère de rationalité qui les entoure de toutes parts.

C'est en poussant à la limite une argumentation de ce genre que l'économiste de tradition autrichienne Ludwig von Mises en est arrivé à mettre l'analyse économique à l'abri de toute attaque possible en soutenant qu'elle dérivait d'un principe de rationalité universellement vrai et vrai $a$ priori. Si tous les individus agissent forcément de manière parfaitement adaptée à la situation telle qu'ils la perçoivent, il est possible d'élaborer déductivement une science de l'action humaine que Mises appelle une "praxéologie" dont la micro-économie constituerait une branche 
particulièrement développée ${ }^{10}$. Seulement, l'ennui de cette approche aprioriste de l'économie, c'est qu'elle enlève au postulat de rationalité une bonne part des avantages qui incitaient à y faire appel. Les économistes trouvent intéressant de postuler que les individus agissent rationnellement dans la mesure où un tel postulat leur permet de prédire leur comportement. Ceci supposait toutefois que les fins poursuivies par ces individus soient relativement stables et qu'elles soient poursuivies par tous les individus d'une catégorie comme, par exemple, celle des entrepreneurs dont il a pu paraître raisonnable de postuler qu'ils ne cherchent qu'à maximiser leurs profits. Si toutefois on admet que ces entrepreneurs peuvent agir au gré de leur fantaisie sans s'écarter pour autant de la rationalité, on perd en possibilité de prédiction ce que l'on gagne en réalisme. Il est vrai qu'il y a, dans le monde réel, des entrepreneurs timorés, superstitieux, maladroits ou hypersensibles qui sont bien loin de prendre toutes les mesures qui seraient susceptibles de maximiser leurs profits. D'un point de vue analogue à celui de von Mises, ils n'agissent pas pour autant de façon irrationnelle dans la mesure où leurs actions sont adaptées aux fins qui sont les leurs au moment où ils agissent. Mais sì l'on sauve, de cette façon, l'universelle validité du postulat de rationalité, peut-on encore espérer, dans ce contexte, arriver à des prédictions scientifiques sérieuses? Et si l'on admet que, plus encore que les entrepreneurs, les membres des autres catégories de la population sont susceptibles de prendre "rationnellement" des décisions fantaisistes et imprévisibles, de quel secours un principe de rationalité valable a priori peut-il être pour le théoricien qui croyait pouvoir y prendre appui afin de prédire le comportement effectif des agents économiques et sociaux? Et si aucune prédiction n'est possible, que reste-t-il de l'espoir de fonder sur ce principe la scientificité des sciences humaines?

Mises et les défenseurs d'une telle conception de la rationalité pouvaient toujours se rabattre sur l'idée que, à moins de s'engager sur le terrain miné des jugements de valeur, la seule rationalité que l'on puisse invoquer en sciences humaines est la rationalité instrumentale, celle qui concerne, non pas les fins elles-mêmes, mais uniquement les moyens d'atteindre des fins déjà données. Dès lors, si l'on refuse par principe de discuter des fins, on ne voit pas comment on pourrait traiter d'irrationnel un entrepreneur qui refuserait de congédier, alors que la situation économique l'exigerait, des employés pour lesquels il se serait pris d'affection ou même un individu qui aurait dépensé la totalité de sa fortune à l'achat

10. Cf. l'introduction de Ludwig von Mises, L'Action humaine, traité d'économie, Paris, Presses universitaires de France, 1985 (trad. de Human Action. A Treatise on Economics, New Haven, Yale University Press, 1949). 
d'une drogue débilitante qui, au moment de sa consommation, lui aurait manifestement procuré une grande satisfaction.

C'est parce qu'une telle façon de voir semble vider de son sens la notion de rationalité que Jon Elster a insisté sur la différence entre une théorie de la rationalité qui s'en tient à ce niveau formel et superficiel (" thin theory ") et une théorie qui, à divers niveaux, permet de discerner ce qui est irrationnel de ce qui est authentiquement rationnel (" broad theory ") ". Alors qu'une théorie formelle ne s'intéressait qu'à t'adaptation d'un comportement à des fins données à la lumière de croyances données, sans discuter ces fins ou ces croyances, une théorie de ce dernier type permettrait d'évaluer la rationalité des fins et des croyances ellesmêmes. Elster s'emploie à analyser des actions de divers types, manifestement déficientes du point de vue de la rationalité, même si, quand on s'en tient à un niveau purement formel, rien n'empêche de les présenter comme des actions orientées vers la satisfaction d'un désir quelconque à la lumière de croyances quelconques. C'est ainsi que le titre de l'un de ses ouvrages, Sour Grapes, renvoie aux raisins prétendument " trop verts " du renard de la fable de La Fontaine dont la réaction motivée par une incapacité plus ou moins refoulée ne peut certes pas être considérée comme particulièrement rationnelle.

Elster a sans doute raison d'insister sur les diverses formes d'irrationalité qui se cachent derrière la rationalité toute formelle des actions qui répondent aux désirs et aux croyances les plus irrationnelles, mais ceci n'implique pas que le principe de rationalité qui joue en sciences humaines le rôle épistémologique examiné ici doive être compris à un niveau autre que le niveau formel de la "thin theory". Dans un ouvrage antérieur, Elster amorçait son analyse de la rationalité et de l'irrationalité en tirant fort ingénieusement parti de l'un des plus célèbres passages de L'Odyssée pour soutenir qu'il y a des actions qui méritent moins que d'autres d'être qualifiées de rationnelles, du fait qu'elles sont plus que d'autres le reflet de la faiblesse de la volonté de leurs auteurs ${ }^{12}$. Ulysse savait qu'il ne pourrait résister à la séduction des sirènes et que cette séduction menaçait gravement son objectif qui était de retourner à Ithaque auprès de Pénélope, mais, en ordonnant à ses marins de l'attacher au mât de son vaisseau, il parvint, par une voie indirecte, à surmonter la faiblesse de sa volonté et, ultimement, à réaliser son objectif. Faut-il, avec Elster, voir dans le fait qu'il ait été ainsi contraint de recourir

11. Voir le premier chapitre de Jon Elster, Sour Grapes. Studies in the Subversion of Rationality, Paris/Cambridge, Maison des sciences de l'homme/Cambridge University Press, 1983 .

12. Ib., Ulysses and the Sirens. Studies in Rationality and Irationality, Paris/Cambridge, Maison des sciences de l'homme/Cambridge University Press, 1979. 
à un tel stratagème le signe d'une faiblesse de volonté qui serait déjà, en elle-même, la manifestation d'une certaine irrationalité, ou, au contraire, voir dans l'astuce avec laquelle il est parvenu à surmonter les contraintes internes et externes qui s'imposaient à lui l'un des triomphes les plus remarquables de la rationalité ? Il me semble que, malgré l'immense intérêt que présentent les analyses de Jon Elster pour une étude psychologique de la volonté et de ses limites, il faut opter pour la seconde réponse quand on s'intéresse plutôt à la question, épistémologique et non psychologique, du contenu du principe de rationalité en sciences humaines. Qu'elle témoigne ou non d'une certaine faiblesse de volonté, l'astuce d'Ulysse sera perçue par quiconque veut tirer parti du principe de rationalité pour comprendre le comportement de ce héros comme une manifestation particulièrement éclatante d'une adaptation des moyens disponibles à des fins qu'il ne s'agit pas alors de discuter, tout comme ne seront pas discutées les croyances et les contraintes psychologiques qui expliquent ce comportement. Ce qui intéresse le spécialiste de L'Odyssée qui recourt au principe de rationalité, ce n'est pas de savoir si Ulysse est un être doté de structures psychologiques parfaitement ou imparfaitement rationnelles, c'est de savoir si, compte tenu tant des fins que des ressources cognitives et psychologiques qu'on lui suppose, son comportement est assez adapté à la situation pour qu'on le considère parfaitement explicable. De même, ce que doit supposer l'économiste, ce n'est pas que l'entrepreneur dispose de la force de caractère, de l'information, voire des ressources technologiques et financières qui seraient requises pour lui permettre d'atteindre ses fins par la voie la plus directe, c'est que l'entrepreneur mettra en ceuvre les moyens dont il dispose pour atteindre ses fins, malgré les contraintes auxquelles il fait face. Bref, le principe de rationalité peut jouer, en sciences humaines, le rôle spécifique qui lui est dévolu dans le mesure où la rationalité dont il est question se comprend dans son sens formel, si limité que soit celui-ci.

PEUT-ON DIRE QUE LE PRINCIPE DE RATIONALTÉ EST UN PRINCIPE EMPIRIQUE?

Ce que garantit un tel principe purement formel, c'est que les actions humaines peuvent être expliquées car elles ne sont jamais absolument gratuites ou absurdes puisqu'elles sont toujours orientées vers la réalisation d'une fin quelconque. Reste à voir cependant s'il faut pour autant en conclure avec Mises que le principe de rationalité est vrai a priori et renoncer, du coup, à en faire l'instrument d'une science empirique. Une 
économie empirique, en effet, ne peut se contenter de reconnaître que toute action est rationnelle au sens de Mises puisqu'elle ne saurait autoriser quelque prédiction que ce soit à l'aide d'un principe de ce genre sans que soient précisées les fins qui sont en cause. Sans doute ne saurait-il être question pour les économistes de s'interroger sur la « rationalité » de ces fins au sens de la "broad theory » d'Elster car ils s'engageraient alors sur un terrain psychologique qui leur est totalement étranger, mais ils n'en doivent pas moins postuler que ces fins sont telles ou telles. On a vu que, traditionnellement, la théorie économique postulait que les agents économiques se donnent pour fin de maximiser leur utilité et que les entrepreneurs, en particulier, se donnent pour fin de maximiser les profits de leur entreprise. Or, comme l'expérience montre qu'il n'en va pas toujours ainsi, de nombreux économistes ont cherché à présenter autrement la signification concrète du principe de rationalité de manière à le rendre plus conforme aux données de l'expérience. Certains ont soutenu que les entrepreneurs ne maximisaient pas forcément leurs profits mais qu'ils cherchaient plutôt à maximiser d'autres variables comme leur prestige, leurs ventes ou leur chiffre d'affaires ${ }^{13}$; mais c'est l'économiste Herbert Simon qui, sur ce plan, a réinterprété le principe de rationalité de la façon la plus décisive. Plutôt que de remplacer la variable à maximiser, H. Simon a proposé de modifier l'idée même d'une maximisation. Pourquoi, en effet, la rationalité exigerait-elle absolument que quelque chose soit maximisé ? N'est-il pas plus raisonnable de supposer que les entrepreneurs cherchent plutôt à obtenir des résultats qu'ils jugent acceptables ou satisfaisants et qu'ils ne réajustent leur tir que dans la mesure où ces résultats tombent en deçà du niveau correspondant à ce satisfecit ${ }^{14}$. Selon Simon, ces entrepreneurs, plutôt que de chercher frénétiquement à maximiser leurs profits, ne prendraient les dispositions qui s'imposent aux yeux des économistes qu'à partir du moment où ils concluent que leurs entreprises respectives ne satisfont plus les critères qui, pour eux, définissent ce qui est acceptable. Et si tel est le cas, pourquoi les actions d'un entrepreneur qui se laisse guider par cette façon de voir les choses ne seraient-elles pas, du point de vue formel qui nous intéresse ici, parfaitement adaptées à ses fins et donc parfaitement rationnelles ? Bien qu'interprété dans un sens que d'aucuns jugeront affaibli, le principe de rationalité ainsi compris constitue une expression qui paraît plus réaliste de ce

13. Voir $\$ 5$, chap. 10, in William J. BAUMOL, Théorie économique et analyse opérationnelle, Paris, Dunod, 1963 (trad. de Economic Theory and Operations Analysis, Englewoods Cliffs, N.J., Prentice Hall, 1961, 1977; dans cette édition, \& 5, chap. 13).

14. Cette thèse souvent reprise par Simon, a d'abord été exposée in Herbert A. Simon, « A Behavioral Model of Rational Choice ", Quarterly Journal of Economics, 69, 1, 1955, p. 99. 118. 
qui, en dernier ressort, guide les entrepreneurs. Ce qu'il importe de bien voir ici, c'est que, sous cette forme " affaiblie ", le principe de rationalité semble sauvé, en ce sens qu'il devient difficile de le rejeter en prétextant qu'il est contredit par l'expérience, mais qu'il n'est pas devenu pour autant un principe a priori comme chez von Mises. Pour peu que soit précisée davantage la notion de satisfaction, on peut espérer à nouveau faire de ce principe la base d'une théorie empirique autorisant de véritables prédictions scientifiques. Pourtant, la partie est loin d'être gagnée, car ou bien la notion de satisfaction est comprise en un sens suffisamment déterminé et le principe risque à nouveau de devoir être considéré comme faux, ou bien elle demeure assez vague et le principe risque à nouveau de perdre son caractère empirique puisque toute action, quelle qu'elle soit, pourrait alors être interprétée comme déclenchée par une chute du niveau de satisfaction sous son seuil minimal.

Même en s'en tenant à la vision toute formelle du principe de rationalité qu'Elster associe à une "thin theory", les théoriciens des sciences humaines ne sauraient se résoudre aisément - la démarche de Simon en témoigne - à renoncer à l'empiricité des disciplines qui y prennent appui. C'est manifestement dans ce contexte que, sir Karl Popper, l'un des plus célèbres épistémologues $\mathrm{du} \mathrm{xx}^{\mathrm{e}}$ siècle, $\mathrm{s}^{\prime}$ est efforcé de concilier rationalité et empiricité dans une tentative dont le moins qu'on puisse dire est qu'elle n'a pas fait l'unanimité dans la communauté épistémologique. Rares, en effet, sont les textes en épistémologie contemporaine qui auront été jugés aussi déconcertants que le petit écrit de moins de dix pages que Popper a consacré au principe de rationalite ${ }^{15}$. Dans un effort pour caractériser le statut de ce principe, auquel il prête un rôle absolument essentiel pour le développement des sciences sociales, Popper y reconnait que ce principe est certainement faux tout en recommandant, à la grande surprise de la plupart de ses lecteurs, de ne pas le traiter comme réfuté pour autant. Plusieurs ont vu là une contradiction flagrante ou, à tout le moins, une ambiguïté fort embarrassante chez un philosophe des

15. Karl POPPER, « La rationalité et le statut du principe de rationalité », in Emil M. ClaAssen, dir., Les Fondements philosophiques des systèmes économiques, textes de Jacques Rueff́ et essais rédigés en son honneur, Paris, Payot, 1967. Ce texte, d'abord paru en traduction française, fut tardivement publié en anglais sous le titre The Rationality Principle (1967), in David Miller, dir., Popper Selections, Princeton, Princeton University Press, 1985. Quoique je citerai ici la version française de ce texte $(R S P R$ ), qui d'ailleurs en a longtemps constitué la seule version disponible, je donnerai la référence aux deux éditions, puisque la version anglaise ( $R P$ ) est de la main de Popper lui-même. Je discute ce texte plus en détail dans M. LAguelx, "Popper et le principe de rationalité ", Cahiers du Dépariement de philosophie, Montreal, Université de Montréal, 9001, 1990; une version anglaise de ce texte doit paraitre sous le titre " Popper and the Rationality Principle " dans un prochain numéro de la revue Philosophy of the Social Sciences. 
sciences dont le nom même est associé au réfutationnisme - c'est-à-dire à la doctrine qui veut qu'une théorie soit scientifique dans la mesure où elle se prête maximalement à une réfutation éventuelle ${ }^{16}$. Le principe de réfutabilité, qui est pourtant au centre de toute l'épistémologie poppérienne, pourrait-il donc être calmement abandonné par son auteur dès qu'il est question de sciences sociales? Il n'est pas surprenant que la démarche de Popper ait suscité des commentaires plutôt négatifs, mais je pense qu'il vaut la peine de s'efforcer de bien la comprendre avant de crier à la contradiction. Même si on risque alors de devoir conclure que cette thèse repose sur des bases passablement fragiles, un tel effort devrait, à tout le moins, aider à mieux cerner la nature exacte du problème posé par le recours au principe de rationalité en sciences humaines.

Dans le contexte de la présente discussion, l'intérêt de la thèse de Popper tient surtout au fait qu'elle constitue une sorte de tentative pour résoudre la quadrature du cercle, c'est-à-dire refuser chacune des options qui, dans la précédente discussion, paraissait épuiser les possibilités offertes à ceux qui entendent ne pas renoncer carrément à recourir au principe de rationalité. Pour Popper, en effet, il n'est pas question d'abandonner ce principe auquel il concédait le rôle central dans la logique situationnelle dont il avait, dans un écrit antérieur ${ }^{17}$, souligné l'importance décisive pour les sciences sociales. Aussi consacre-t-il le petit texte qui nous intéresse ici à défendre le principe de rationalité qu'il désigne volontiers du nom de « principe de l'action adaptée à la situation ${ }^{18}$ en lui donnant la formulation "personnelle» suivante : "les individus agissent toujours d'une manière adaptée à la situation où ils se trouvent $"{ }^{19}$. Sans doute aurait-il pu, à la manière de von Mises, recourir à un principe dépourvu de tout contenu empirique (un principe qui affirme, par exemple, que quoi que fassent les individus, ils le font forcément en vue d'améliorer a leurs propres yeux leur situation, en agissant d'une façon qu'ils estiment alors appropriée, compte tenu de l'état psy-

16. Voir, par ex., Douglas Wade Hands, « Karl Popper and Economic Methodology. A New Look ", Economics and Philosophy, 1, 1, 1985, p. 83-99. Pour une interprétation du texte de Popper fort différente à la fois de celle de Hands et de celle proposée ici, on pourra lire Robert Nadeau, "Confuting Popper on the Rationality Principle », Cahiers d'épistémologie, Montréal, Université du Québec à Montréal, 9012, 1990; ce texte doit paraître dans un prochain numéro de la revue Philosophy of the Social Sciences.

17. K. Popper, Misère de lhistoricisme, Paris, PJon, 1956; trad. franç. (remaniée et augmentée) de trois articles parus sous le titre a The Poverty of Historicism " dans la revue Economica, vol. 11, 1944, p. 86-103, vol. 11, 1944, p. 119-137 et vol. 12, 1945, p. 69-89; une version anglaise également remaniée est parue après la version française : The Poverty of Historicism, Londres, Routledge and Kegan Paul, 1957.

18. C. art. cit. supra n. $15, R S P R$, p. $146 ; R P$, p. 361.

19. Ibid., RSPR, p. 145 ; RP, p. $36 \mathrm{I}$. 
chologique où ils se trouvent), mais, du coup, il aurait prêté le flanc à une accusation d'apriorisme, ce qu'en bon empiriste il redoutait par-dessus tout. Aussi Popper a-t-il tenu à prendre tout particulièrement ses distances à l'égard d'une position comme celle de von Mises et à dénoncer explicitement ceux qui prétendent que le principe de rationalité « est $a$ priori valide ou a priori vrai $"{ }^{20}$. Loin de laisser entendre que le principe de rationalité pourrait être vrai $a$ priori, Popper va même jusqu'à assurer qu'un tel principe est manifestement faux.

Comment alors Popper pouvait-il recommander de ne pas rejeter un principe qu'il qualifiait ainsi de faux? Pour essayer de le comprendre, observons tout de suite que si Popper nous assure avec la dernière énergie que ce principe est faux, il soutient également, à différentes reprises, que ce principe demeure néanmoins à ses yeux une «bonne approximation » de la vérité ${ }^{21}$. Si Popper tient ce principe pour faux, c'est au nom d'une sorte de purisme épistémologique qui ne doit pas nous leurrer sur la signification du verdict qui l'amène à le déclarer "faux » : " un principe qui n'est pas universellement vrai est faux. Par conséquent, le principe de rationalité est faux $"{ }^{22}$. Bref, en soutenant que le principe est faux, Popper signifie qu'il y a effectivement certains cas où ce principe est contredit par les faits, même si, à ses yeux, ces cas sont peu représentatifs du comportement humain.

Popper n'ignorait évidemment pas combien la bêtise, la folie et la névrose sont monnaie courante pour quiconque s'intéresse un peu à la psychologie humaine. Pourtant, explique-t-il sur ce point, il ne faudrait pas en conclure trop vite à l'irrationalité car Freud aurait montré que le névrosé, même quand il crée littéralement son problème, répond de façon tout à fait adaptée à sa situation telle qu'il la voit ${ }^{23}$. Reste que s'il entendait demeurer empiriste et éviter de glisser imperceptiblement vers l'apriorisme de von Mises - pour lequel toute action, si folle qu'elle puisse paraître, est forcément rationnelle dans la mesure où elle est adaptée à la situation telle que la " voit " son auteur -, Popper devait fixer un cran d'arrêt au-delà duquel il ne serait plus raisonnable de dire qu'une action est rationnelle. Pour bien voir où il choisit de situer ce "cran d'arrêt ", le plus simple est de rapprocher deux exemples auxquels il se réfère dans son texte, empruntés d'ailleurs tous deux au monde de la circulation. Pour illustrer en quel sens le comportement du névrosé peut

20. Ibid., RSPR, p. $145 ;$ RP, p. 360.

21. Ibid., RSPR, p. 148, voir aussi p. 147; RP, p. 364 et p. 363.

22. Ibid., RSPR, p. $145 ; R P$, p. 361 .

23. Ibid., RSPR, p. $148 ; R P$, p. 363-364; l'expression en italique est employée à propos de l'exemple des chefs de guerre incompétents, lequel vise à établir la meeme thèse, RSPR, p. $147 ; R P$, p. $362-363$. 
être qualifié de rationnel, Popper le compare à celui d'un piéton qui, pour éviter une auto, se jette sur une voie parallèle où il sera heurté par un cycliste ${ }^{24}$. S'il avait mieux $v u$, le piéton aurait sans doute pu éviter les deux accidents; mais, compte tenu de ce qu'il voyait, son esquive, tout comme l'action du névrosé qui, lui, ne voit que certains aspects de sa situation, constitue une réponse parfaitement adaptée et donc parfaitement rationnelle.

Par contre, pour montrer que l'on rencontre à l'occasion des comportements inadaptés, Popper soutient que, par exemple, un automobiliste énervé qui s'adonnerait à des manceuvres inutiles pour se garer dans un espace visiblement trop exigu ${ }^{25}$ n'agirait manifestement pas de manière appropriée à la situation dans laquelle il se trouve. Bref, cet automobiliste énervé se laisserait aller à des gestes qui ne seraient même pas adaptés à la situation telle qu'il la voit. Quelle différence si fondamentale y aurait-il donc entre le geste du piéton menacé et celui de l'automobiliste énervé? Cette différence saute d'autant moins aux yeux que Popper ne tente nullement ni d'analyser plus avant ni même de rapprocher ces deux exemples. Pour faire ressortir cette différence, il faut supposer que Popper prend le verbe " voir" dans son sens littéral ou, en tout cas, dans son sens strictement cognitif. L'automobiliste verrait bien qu'il ne peut introduire sa voiture dans un aussi petit espace, mais il serait si profondément enragé qu'il aurait perdu tout contrôle et se laisserait littéralement aller à des mancuvres désespérées comme pour y parvenir malgré tout, en s'obligeant par-là à sortir péniblement ensuite de l'espace exigu où il s'est enfoncé bien inutilement. Selon Popper, on ne retrouverait pas là le caractère rationnel qu'il reconnaissait au mouvement du piéton: celui-ci était certes incapable de voir autre chose que l'automobile qui risquait de le heurter, mais dans le cadre de ce qui lui était donné de voir, il a su agir de la façon qui lui paraissait la plus adaptée, c'est-à-dire la plus susceptible de le mettre à l'abri de la collision qui le menaçait

Sans doute l'analyse psychologique de Popper est-elle un peu courte. II serait facile, par exemple, de chercher dans les perceptions de l'automobiliste énervé des éléments qui permettraient d'affirmer que son comportement est adapté, lui aussi, ne serait-ce qu'à un vague besoin de se défouler. Appelé à se justifier, cet automobiliste aurait peut-être répondu : " ça m'a fait du bien de montrer jusqu'à quel point tout devient absurde quand on cherche à se garer dans une ville aussi mal administrée!" Mais l'analyse psychologique de Popper n'a manifeste-

24. Ibid., $R S P R$, p. $148 ; R P$, p. 364.

25. Ibid, $R S P R$, p. $145 ; R P$, p. 361. 
ment pas fait place à ce genre de raffinement. Pour Popper, le névrosé, le piéton et l'automobiliste ne " voient "strictement que les divers éléments caractérisant leur situation respective. Popper ne semble pas vouloir inclure dans les éléments caractérisant cette situation l'état psychologique de chacun (par exemple, le besoin de se défouler) et la façon dont cet état psychologique est subjectivement perçu. Il semble supposer que l'automobiliste voit bien que l'espace est trop petit et qu'il ne peut que perdre un temps précieux en s'adonnant à des manceuvres inutiles. Mais plutôt que de supposer que cet automobiliste ne renonce pas pour autant à ces manœuvres insensées, parce que sa frustration lui fait "voir » les choses autrement, Popper préfère conclure qu'il agit d'une façon bêtement irrationnelle et que, de ce fait, son comportement réfute la validité universelle qu'on aurait pu être tenté d'attribuer au principe de rationalité.

Chacun reconnaitra que la frontière entre les deux types de situation (celle de l'automobiliste énervé et celle du piéton menacé d'être heurté) est beaucoup plus difficile à tracer que ne le suppose Popper. En cherchant à juger objectivement le degré de rationalité des comportements, celui-ci s'engage manifestement dans des difficultés insurmontables qu'il devait sûrement entrevoir. Pourtant, Popper n'ayant jamais fait montre d'une finesse exceptionnelle en matière d'analyse psychologique, je crois qu'il est préférable de mettre en cause la qualité de son analyse sur ce plan plutôt que de prétendre qu'il se soit contredit de façon flagrante en matière épistémologique où il est habituellement si rigoureux. Une telle conclusion ne saurait être que renforcée quand on considère que, en l'espace de quelques pages qu'il n'a jamais répudiées par la suite ${ }^{26}$, Popper reformule à plusieurs reprises la thèse qui serait responsable de cette contradiction.

Reste que cette thèse soulève d'autres problèmes dont le plus manifeste est celui posé par son refus de rejeter, malgré ses convictions réfutationnistes, un principe dont il admettait si volontiers la fausseté. Il faut reconnaitre que, sur ce point, sa réponse est un peu trop sommaire. Elle se développe en quelques brefs arguments qui visent tous à montrer que, dans les cas où le principe de rationalité se trouve contredit, il est à la fois plus raisonnable et plus avantageux, pour le progrès de la connaissance, de tenir pour responsables les autres éléments du modèle dans le cadre duquel ce principe joue le rôle central plutôt que de rejeter le principe de rationalité lui-même ${ }^{27}$. On pourrait penser qu'en proposant ainsi de maintenir ce principe "faux » au nom, en somme, de son utilité cogni-

26. Il a même publié son texte en anglais, dix-huił ans plus tard, sans chercher à le modifier le moindrement, voir à ce sujet, supra n. 15.

27. Cf. art. cit. stupra n. 15, RSPR, p. $147 ; R P$, p. 362. 
tive, Popper lui attribue une fonction purement instrumentale et rejoint l'option dite instrumentaliste qui consistait, on l'a vu, à ne pas se préoccuper de la fausseté d'un principe dans la mesure oì il constitue un bon instrument de prédiction. Ce serait pourtant se méprendre sur l'attitude de Popper, qui a toujours été aussi farouchement opposé à l'instrumentalisme qu'à l'apriorisme ${ }^{28}$. Si le principe de rationalité est pour lui un instrument, celui-ci est ici au service de la compréhension des phénomènes sociaux et non au service du succès de simples prédictions. Bien loin de remettre en cause la primauté de son idéal cognitif, c'est au nom du progrès de la connaissance qu'il propose de maintenir, malgré sa fausseté, un principe qu'il juge indispensable à ce progrès du savoir. D'ailleurs, c'est dans un esprit tout à fait contraire à celui de l'instrumentalisme que Popper insiste pour dire que le principe de rationalité constitue une si bonne approximation de la vérité qu'il est bien peu probable que l'échec d'un test, qui serait susceptible de réfuter la théorie, puisse lui être exclusivement imputé. Bref, en le qualifiant de «faux », Popper reconnaît sans détour les limites du principe de rationalité, mais il n'en assure pas moins que, dans la mesure où des phénomènes sociaux peuvent être expliqués, ils ne peuvent l'être qu'à l'aide de cet indispensable principe.

LE PRINCIPE DE RATIONALTEE COMME CONDITION D'INTELLIGIBILITÉ

Il ne s'agit pas ici de donner raison ou tort à Popper à propos du principe de rationalité, mais de voir comment sa démarche embarrassée reflète mieux que toute autre peut-être l'ambiguïté du statut du principe de rationalité en sciences sociales. Ce principe qui est requis pour qu'un phénomène puisse être expliqué, ou mieux compris, dans le champ des sciences humaines doit, somme toute, être considéré comme une sorte de condition d'intelligibilité. Dans la mesure où un phénomène social dérive d'actions humaines, il sera, en un sens, expliqué si on parvient à en rendre compte à partir d'actions humaines elles-mêmes compréhensibles; or, une action humaine n'est compréhensible que si elle respecte le principe de rationalité compris dans son sens le plus large (celui dont traite la "thin theory" d'Elster). Toutefois, cet état de choses ne saurait garantir a priori que toutes les actions humaines sont compréhensibles. Les sciences sociales se heurtent à de telles difficultés que rien n'empêche

28. Popper est, en effet, l'un des adversaires les plus notoires de la thèse instrumentaliste; cf. Karl R Popper, Conjectures and Refutations. The Growth of Scientific Knowledge, New York, Harper \& Row, 1962, 1965, chap. 3, \& 5. 
de penser que certaines de celles-ci soient dues au caractère rigoureusement incompréhensible de certaines actions humaines. On peut donc imaginer que ces dernières échapperaient absolument au principe de rationalité et c'est ce que Popper a tenté de faire - de façon fort peu convaincante à vrai dire - à l'aide de l'exemple de l'automobiliste énervé. Quoi qu'il en soit de l'existence de cas plus convaincants que celui invoqué par Popper, les explications proposées par ces sciences ne peuvent évidemment être acceptées a priori et doivent être soumises à des tests empiriques qui risquent cependant de porter beaucoup plus sur les divers éléments d'un modèle explicatif que sur le principe de rationalité qui, pour employer une expression de Popper, "anime " en quelque sorte ce modèle ${ }^{29}$. S'efforcer de comprendre un geste qui parait absurde comme celui de l'automobiliste, c'est décider méthodologiquement de recourir à ce principe puisque toute autre attitude équivaudrait à renoncer à comprendre, mais ce n'est pas le tenir pour vrai car rien n'empêche de penser que certaines actions humaines, qui alors demeureraient inexplicables, n'échappent pas à son emprise. En ce sens, le principe de rationalité, sans pouvoir être dit vrai a priori, peut effectivement être considéré comme une sorte de principe d'intelligibilité qu'il n'est jamais souhaitable de traiter comme un principe réfuté.

Au terme de ce parcours, il parait plus facile de se convaincre du fait que le principe de rationalité est inséparable de la notion même de sciences sociales que de définir son statut original de façon vraiment satisfaisante. Aussi ne faut-il pas s'étonner de voir que, dans diverses sciences sociales, on ait eu recours à un principe de ce genre, tout en se gardant le plus souvent d'endosser explicitement une épistémologie qui en préciserait le rôle central. C'est pour cela sans doute que l'option instrumentaliste, qui dispense de se préoccuper de la fonction pourtant incontournable impartie au principe de rationalité, est apparue beaucoup plus séduisante aux économistes que les tentatives peu gratifiantes qui visaient à préciser le statut de ce principe. En économie, seule la tradition autrichienne a fortement mis l'accent sur l'idée qu'il importerait moins, pour l'analyse économique, de prédire des phénomènes à l'aide de prétendues lois économiques que de comprendre pourquoi, compte tenu des objectifs qu'il paraît raisonnable d'imputer à des agents présumés rationnels, ces phénomènes se sont développés de telle ou telle façon. Si l'on estime que le rôle d'une science sociale est de comprendre après coup des phénomènes sociaux qui, sans cette explication, auraient risqué de demeurer étranges et souvent mystérieux, alors un principe qui assure

29. Cf. art. cit. supra n. $15, R S P R$, p. 143 ; RP, p. 358-359. 
que les individus agissent en vue de réaliser des fins qui leur sont propres, si vide qu'il puisse paraître au premier abord, devient déterminant.

On reconnaitra que cette façon de comprendre le rôle des sciences humaines et des sciences sociales en particulier n'intéresse évidemment pas que l'économie. Aussi n'est-il pas étonnant que l'on ait retrouvé - à propos de la sociologie, dans le monde germanique, avec les controverses portant sur la notion de Verstehen ${ }^{30}$ et, à propos de l'histoire, dans le monde anglo-saxon, avec les controverses entourant la notion de Covering $l a w^{31}$ - des débats largement parallèles à celui qui, à propos de l'économie, concerne cette conception de l'intelligibilité souvent associée à la tradition «autrichienne ». Malgré des différences que je m'en voudrais de chercher à dissimuler, dans chacun de ces cas, la question principale est celle de savoir s'il y a place pour une science qui permet d'expliquer (au sens de « comprendre », de « déchiffrer », ou mieux de « rendre intelligible ») ce qui s'est passé dans un secteur donné de l'univers extrêmement complexe des phénomènes sociaux sans que, pour autant, la validité de ces explications soit cautionnée, comme c'est généralement le cas en sciences physiques, par le succès de prédictions qu'elles autoriseraient. Si le statut épistémologique du principe de rationalité, au cœur d'une telle entreprise, paraît aussi difficile à cerner, c'est parce qu'on ne peut se satisfaire d'une analyse épistémologique qui réserve à ce principe un rôle sans équivalent dans les sciences physiques à partir desquelles ont été façonnées nos catégories épistémologiques. Mais si l'on accepte l'idée que les sciences humaines diffèrent des sciences physiques, c'est à n'en pas douter en ceci que quelque chose comme un principe de rationalité est requis pour rendre compte des actions humaines à l'origine des phénomènes sociaux. C'est la place qu'on a réservée à ce principe difficilement éliminable dans une science sociale en particulier, soit la science économique, que le présent article a voulu mettre en lumière. Il aura atteint son but si l'examen des diverses positions adoptées a pu faire ressortir quelque peu, non seulement le caractère ambigu du statut qu'il y a lieu de reconnaître à un tel principe, mais aussi le caractère spécifique du processus qui a permis à certaines disciplines de progresser en y prenant appui.

Maurice Lagueux, Université du Québec à Montréal.

30. On relira, à ce sujet, l'étude classique de Raymond Aron, La Philosophie classique de l'histoire. Essai sur une théorie allemande de l'histoire, Pris, Vrin, 1970.

31. Faute de pouvoir donner un aperçu sérieux des multiples interventions dans ce débat associé surtout aux noms de Carl Hempel et de William Dray, je me contenterai de signaler un ouvrage très récent qui en fait état en le situant dans une perspective plus large : Alain BOYER, L'Explication en histoire, Lille, Presses universitaires de Lille, 1992. 